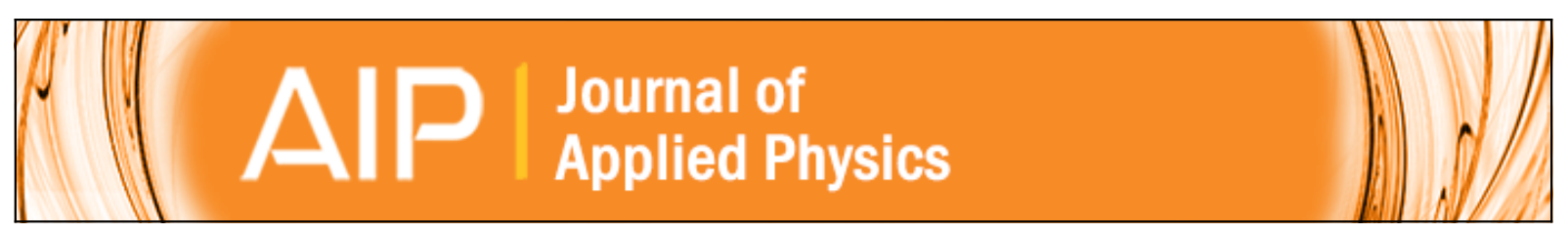

\title{
Phonon anomalies and superconductivity in the Heusler compound YPd2Sn
}

H. M. Tütüncü and G. P. Srivastava

Citation: Journal of Applied Physics 116, 013907 (2014); doi: 10.1063/1.4887355

View online: http://dx.doi.org/10.1063/1.4887355

View Table of Contents: http://scitation.aip.org/content/aip/journal/jap/116/1 ?ver=pdfcov

Published by the AIP Publishing

\section{Articles you may be interested in}

Superconductivity at $28 \mathrm{~K}$ in $\mathrm{CaB} 3 \mathrm{C} 3$ predicted from first-principles

J. Appl. Phys. 114, 173906 (2013); 10.1063/1.4829458

Theoretical examination of superconductivity in the cubic antiperovskite $\mathrm{Cr} 3 \mathrm{GaN}$ under pressure J. Appl. Phys. 114, 053905 (2013); 10.1063/1.4817072

First-principles prediction of layered antiperovskite superconductors $\mathrm{A} 2 \mathrm{CNi} 4(\mathrm{~A}=\mathrm{Al}, \mathrm{Ga}$, and $\mathrm{Sn}$ ) AlP Advances 2, 042167 (2012); 10.1063/1.4769882

Phonons and superconductivity in the cubic perovskite Cr3RhN

J. Appl. Phys. 112, 093914 (2012); 10.1063/1.4764916

Ab initio investigations of phonons and superconductivity in the cubic Laves structures LaAl2 and YAl2 J. Appl. Phys. 111, 033514 (2012); 10.1063/1.3681327

\section{A|P| Journal of}

Journal of Applied Physics is pleased to announce André Anders as its new Editor-in-Chief 


\title{
Phonon anomalies and superconductivity in the Heusler compound $\mathrm{YPd}_{\mathbf{2}} \mathrm{Sn}$
}

\author{
H. M. Tütüncü ${ }^{1}$ and G. P. Srivastava ${ }^{2}$ \\ ${ }^{1}$ Sakarya Üniversitesi, Fen-Edebiyat Fakültesi, Fizik Bölümü, 54187, Adapazarl, Turkey \\ ${ }^{2}$ School of Physics, University of Exeter, Stocker Road, Exeter EX4 4QL, United Kingdom
}

(Received 6 May 2014; accepted 25 June 2014; published online 7 July 2014)

\begin{abstract}
We have studied the structural and electronic properties of $\mathrm{YPd}_{2} \mathrm{Sn}$ in the Heusler structure using a generalized gradient approximation of the density functional theory and the $a b$ initio pseudopotential method. The electronic results indicate that the density of states at the Fermi level is primarily derived from Pd d states, which hybridize with Y d and Sn p states. Using our structural and electronic results, phonons and electron-phonon interactions have been studied by employing a linear response approach based on the density functional theory. Phonon anomalies have been observed for transverse acoustic branches along the [110] direction. This anomalous dispersion is merely a consequence of the strong coupling. By integrating the Eliashberg spectral function, the average electron-phonon coupling parameter is found to be $\lambda=0.99$. Using this value, the superconducting critical temperature is calculated to be $4.12 \mathrm{~K}$, in good accordance with the recent experimental value of 4.7 K. (C) 2014 AIP Publishing LLC. [http://dx.doi.org/10.1063/1.4887355]
\end{abstract}

\section{INTRODUCTION}

Heusler compounds have attracted a great deal of attention in recent years since they are covenanting for several handy applications in the field of smart materials and spintronics. ${ }^{1-7}$ It should be mentioned that the area of spintronics is one of the most quickly broadening areas of nanoscience and technology since the incorporation of the electron's spin provides an additional degree of freedom to be used for information processing in nanodevices. ${ }^{8}$ Outside of spintronics, Heusler compounds with high spin polarization, high Curie temperature and concurrently a low saturation magnetization are convenient for ultrahigh density magnetic memory storage devices. ${ }^{7}$ In addition to their magnetic properties, superconductivity was discovered for several Heusler compounds. In 1982, superconductivity was discovered for the cubic Heusler compound $\mathrm{YPd}_{2} \mathrm{Sn}$ with the transition temperature of 3.72 in the experimental work of Ishikawa. ${ }^{9}$ This discovery has motivated experimentalists to investigate its structural and superconducting properties. In 1984, Johnson and Shelton ${ }^{10}$ observed that the application of hydrostatic pressure linearly depressed the superconducting transition temperature with a typical value of $\frac{d T_{C}}{d P} \sim 10^{-5} \mathrm{~K} / \mathrm{bar}$ for the Heusler compounds $\mathrm{RPd}_{2} \mathrm{X}(\mathrm{R}=\mathrm{Sc}, \mathrm{Y}, \mathrm{Tm}, \mathrm{Yb}$, and $\mathrm{Lu}$ and $\mathrm{X}=\mathrm{Sn}, \mathrm{Pb}$ ). Following this study, Malik and co-workers showed that the Heusler compound $\mathrm{YPd}_{2} \mathrm{Sn}$ is superconducting with a transition temperature of $4.55 \mathrm{~K}^{11,12}$ Heat-capacity measurement ${ }^{13}$ indicated that the transition temperature of this Heusler compound is between 5.04 and $5.26 \mathrm{~K}$. Recently, the lattice parameter of $\mathrm{YPd}_{2} \mathrm{Sn}$ has been measured in the experimental work of Höting and coworkers. ${ }^{14}$ Superconducting properties of $\mathrm{YPd}_{2} \mathrm{Sn}$ have been presented in the experimental work of Klimczuk et al. ${ }^{15}$ Following this work, Saadaoui and co-workers ${ }^{16}$ have reported on muon-spin rotation and relaxation $(\mu \mathrm{SR})$ and ${ }^{119} \mathrm{Sn}$ nuclear magnetic resonance (NMR) measurements to study the microscopic superconducting and magnetic properties of the Heusler compound $\mathrm{YPd}_{2} \mathrm{Sn}$. Although considerable improvement has been done in experimental explanation of the superconducting properties of the Heusler compound $\mathrm{YPd}_{2} \mathrm{Sn}$, no systematic theoretical attempt has been made to investigate structural, electronic, and superconducting properties of this material so far. Such studies must be done, since phonons play the role of bringing about the coupling between electrons to form Cooper pairs, which are amenable for superconductivity, as explained in the BCS theory.

In this paper, we have carried out $a b$ initio calculations of the ground state and electronic properties of $\mathrm{YPd}_{2} \mathrm{Sn}$ in the Heusler structure. The electronic properties are investigated by using the plane wave pseudopotential method within the generalized gradient approximation of the density functional scheme. This is followed by the application of a linear response scheme ${ }^{17}$ for the calculation of phonon dispersion curves and phonon density of states. Atomic displacement patterns and electron phonon coupling parameters for zone-centre phonon modes have been presented and discussed in detail. Furthermore, the Eliashberg spectral function and the average electron-phonon coupling constant $\lambda$ are calculated. We have compared the Eliashberg spectral function with the corresponding phonon density of states (DOS) in detail. This comparison shows that Pd-related phonon modes make a large contribution to the average electronphonon coupling constant as a result of the considerable existence of Pd d electrons at the Fermi energy. Using the calculated value of $\lambda$, the superconducting critical temperature and the electronic specific heat coefficient $\gamma$ are calculated. Finally, the calculated superconducting parameters are favourably compared with their corresponding values in the recent experimental work of Klimczuk and co-workers. ${ }^{15}$

\section{THEORY}

The QUANTUM ESPRESSO code ${ }^{17}$ employs a planewave basis set of the expansion of the Kohn-Sham orbitals. ${ }^{18}$ The exchange-correlation energy of electrons is defined in the generalized gradient density functional approximation (GGA) 
using the Perdew-Burke-Erzerhof expression. ${ }^{19}$ The electronion interaction is described by using norm-conserving pseudopotentials. $^{20,21}$ The maximum plane wave cutoff energy is taken as 60 Ry while the electronic charge density is expanded in a basis cut off up to $240 \mathrm{Ry}$. Self-consistent solutions of the Kohn-Sham equations ${ }^{18}$ were obtained by employing a set of Monkhorst-Pack special $\mathbf{k}$ points ${ }^{22}$ within the Brillouin zone. The numerical integration of the Brillouin zone (BZ) for the total-energy calculations is performed by using a $16 \times 16 \times 16$ Monkhorst-Pack k-point sampling. ${ }^{22}$ On the other hand, the k-point mesh that is used for the electronic calculations in the $\mathrm{BZ}$ is chosen as $32 \times 32 \times 32$.

The Monkhorst-Pack $16 \times 16 \times 16 \mathbf{k}$-points grid within the $\mathrm{BZ}$ is used for the phonon calculations. We calculate the phonon frequencies and atomic displacements using the linear response method, ${ }^{17}$ which avoids the use of supercells and allows calculation of the dynamical matrix at arbitrary phonon $\mathbf{q}$ vectors. A static linear response of the valence electrons is taken into account in terms of the variation of the external potential corresponding to periodic displacements of the atoms in the unit cell. The screening of the electronic system in response to the displacement of the atoms is considered in a self consistent manner. Twenty nine dynamical matrices are calculated using a mesh of $(8,8,8)$ reciprocal lattice divisions in order to obtain full phonon dispersion relations. These dynamical matrices are Fourier transformed to obtain the full phonon spectrum and density of states. We estimate that the phonon frequencies are accurate to within $0.1 \mathrm{THz}$ for the present choice of the kinetic energy cutoff and the special $\mathbf{k}$ points.

The GGA electronic calculations together with the linear response method for the determination of the phonon dispersion relations, electron-phonon coupling, and solution of the Eliashberg equations have made it possible to obtain transition temperatures and study in detail electron-phonon interaction. The Eliashberg electron-phonon spectral function $\alpha^{2} F(\omega)$ can be presented as ${ }^{23-25}$

$$
\alpha^{2} F(\omega)=\frac{1}{2 \pi N\left(E_{F}\right)} \sum_{\mathbf{q} j} \frac{\gamma_{\mathbf{q} j}}{\hbar \omega_{\mathbf{q} j}} \delta\left(\omega-\omega_{\mathbf{q} j}\right),
$$

where $\mathrm{N}\left(\mathrm{E}_{F}\right)$ is the electronic density of states at the Fermi level and $\gamma_{\mathbf{q} j}$ indicates the phonon line-width. When the electron energies around the Fermi level are linear in the range of phonon energies, the phonon line-width is given by the Fermi's "golden rule" formula ${ }^{24,25}$

$$
\gamma_{\mathbf{q} j}=2 \pi \omega_{\mathbf{q} j} \sum_{\mathbf{k} n m}\left|\mathrm{~g}_{(\mathbf{k}+\mathbf{q}) m ; \mathbf{k} n}^{\mathbf{q} j}\right|^{2} \delta\left(\varepsilon_{\mathbf{k} n}-\varepsilon_{F}\right) \delta\left(\varepsilon_{(\mathbf{k}+\mathbf{q}) m}-\varepsilon_{F}\right),
$$

where the Dirac delta functions express energy conservation conditions, and $g$ is the electron-phonon matrix element. Finally, we are able to determine the electron-phonon coupling parameter $(\lambda)$ From $\alpha^{2} F(\omega)$, which is a good measure of the overall strength of the electron-phonon interaction; it is given by

$$
\lambda=\int \lambda(\omega)=2 \int \frac{\alpha^{2} F(\omega)}{\omega} d \omega .
$$

The summations in Eqs. (1) and (2) are made using a dense mesh $((32 \times 32 \times 32)$ Monkhorst-Pack mesh $)$ of $\mathbf{k}$ points in the BZ. The Dirac delta functions in this equation are replaced with a Gaussian function of width $0.01 \mathrm{Ry}$.

\section{RESULTS}

\section{A. Structural and electronic properties}

$\mathrm{YPd}_{2} \mathrm{Sn}$ adopts the cubic Heusler structure with the space group Fm $\overline{3} \mathrm{~m}$ (see Fig. 1(a)), which includes one formula unit in the primitive unit cell with Wyckoff positions of atoms at $\mathrm{Y}(4 \mathrm{~b})\left(\frac{1}{2}, \frac{1}{2}, \frac{1}{2}\right), \mathrm{Pd}(8 \mathrm{c})\left(\frac{1}{4}, \frac{1}{4}, \frac{1}{4}\right)$, and Sn (4a) $(0,0,0)$. For the calculation of the ground state properties such as the equilibrium lattice constant $a$, the bulk modulus $\mathrm{B}$, and its pressure derivative $\mathrm{B}^{\prime}$, we have calculated the total energy as a function of lattice constant. The obtained relationship between total energy and lattice constant is displayed in Fig. 1(b). The calculated total energies are fitted to the Murnaghan's equation of state ${ }^{26}$ to determine the values of the ground state properties. The calculated bulk static properties from our total energy calculations are presented in Table I together with available experimental results. In comparison with the experimental data, we have found that the

(a)
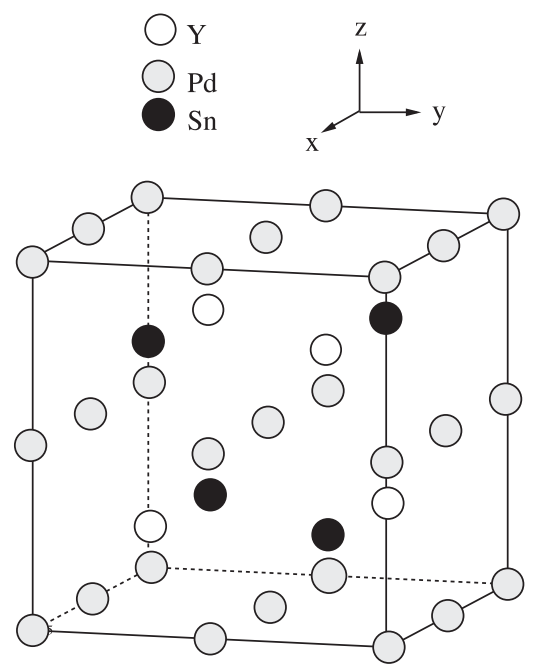

(b)

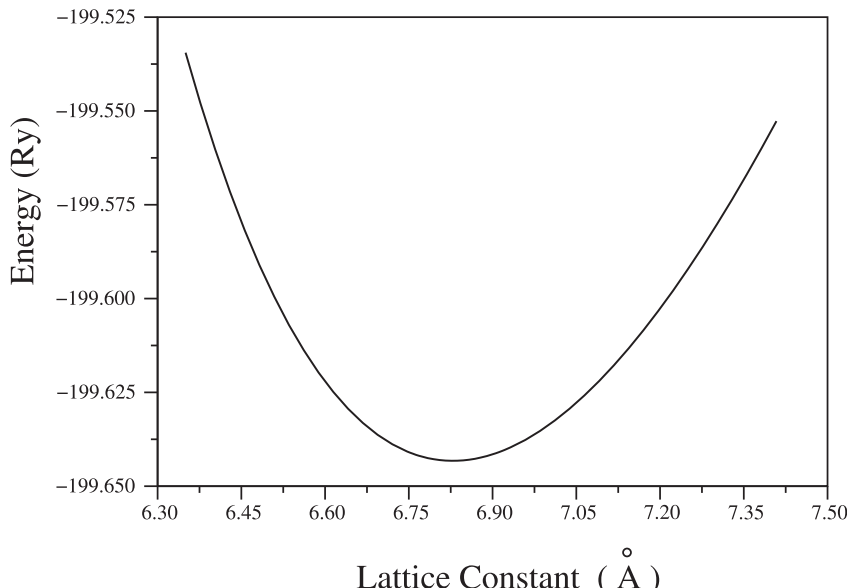

FIG. 1. (a) The cubic Heusler structure of $\mathrm{YPd}_{2} \mathrm{Sn}$. (b) Calculated total energies as a function of lattice constant. 
TABLE I. Static properties of the superconducting Heusler compound $\mathrm{YPd}_{2} \mathrm{Sn}$ and their comparison with previous experimental and theoretical results.

\begin{tabular}{lccc}
\hline \hline Source & $a(\AA)$ & B (Mbar) & B' \\
\hline This study(GGA) & 6.8289 & 1.004 & 4.70 \\
Experimental $^{9,10}$ & 6.7190 & 1.073 & \\
Experimental $^{11,12}$ & 6.7160 & & \\
Experimental $^{13}$ & 6.7165 & & \\
Experimental $^{14}$ & 6.7144 & & \\
Experimental $^{15}$ & 6.7160 & & \\
\hline \hline
\end{tabular}

calculated lattice constant is slightly larger than the measured ones with around $1.7 \%$. The calculated bulk modulus (B) deviates from its experimental value of $1.073 \mathrm{Mbar}$ within $6 \%$. To our knowledge, there are no other theoretical calculations for the ground state properties of $\mathrm{YPd}_{2} \mathrm{Sn}$ in the literature, so more theoretical calculations should be made for comparison.

The calculated energy band structure, at the equilibrium lattice constant for $\mathrm{YPd}_{2} \mathrm{Sn}$, along the high symmetry directions in the Brillouin zone is shown in Fig. 2. The analysis of the electronic structure indicates the metallic nature of this material because conduction and valence bands cross the Fermi level. The lowest band in this figure lying between 9.0 and $7.5 \mathrm{eV}$ below the Fermi level is dominated by the hybridization of Sn 5s and Pd $4 \mathrm{p}$ states. This band is well separated from other bands by a gap of $2.5 \mathrm{eV}$. This band has a parabolic shape along the main symmetry directions of $\Gamma-\mathrm{K}$, $\Gamma-\mathrm{X}$, and $\Gamma$-L while it is totally flat along other symmetry directions. The next group of occupied bands is from a mixture of $\mathrm{Pd}, \mathrm{Y}$, and $\mathrm{Sn}$ orbitals. In particular, we have observed a flat conduction band at approximately $0.15 \mathrm{eV}$ above the Fermi level along the $\Gamma-\mathrm{X}$ and $\Gamma$-L symmetry directions. This feature is very important for the superconducting properties of $\mathrm{YPd}_{2} \mathrm{Sn}$ since electrons near the Fermi surface are strongly involved in the formation of the superconducting state.

The total and partial DOS for $\mathrm{YPd}_{2} \mathrm{Sn}$ are provided in Fig. 3. The total DOS has remarkable features with energies of $-7.8,-4.8,-4.1,-3.3,-2.8$, and $-1.1 \mathrm{eV}$. The first

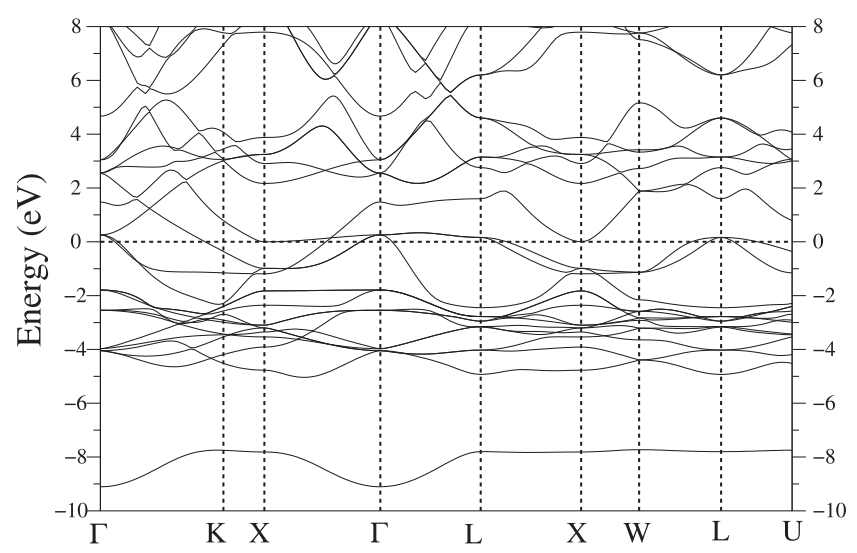

FIG. 2. Electronic band structure for $\mathrm{YPd}_{2} \mathrm{Sn}$ along the high symmetry lines of the Brillouin zone. Zero energy represents the Fermi level.
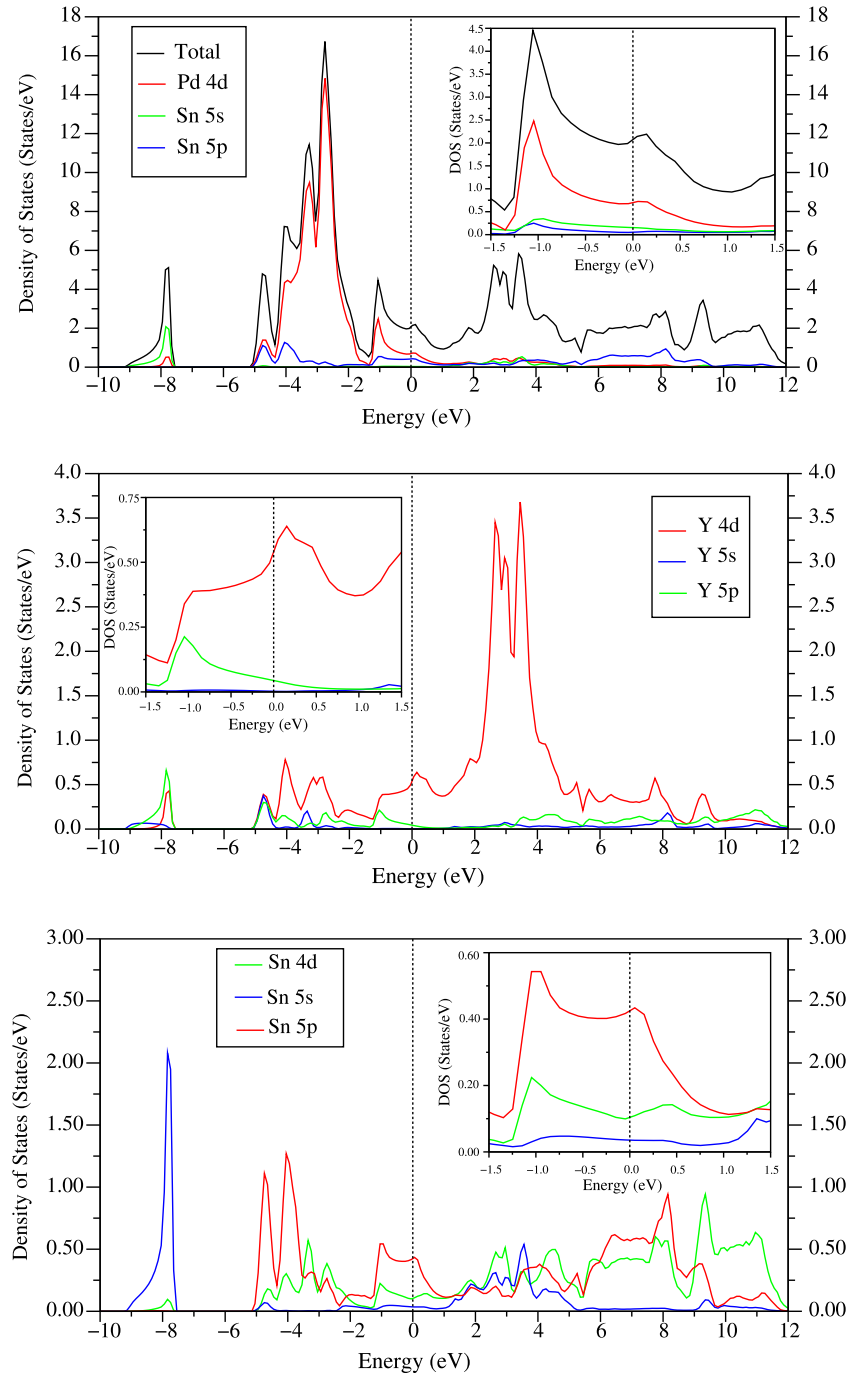

FIG. 3. Total and partial density of states for $\mathrm{YPd}_{2} \mathrm{Sn}$. The Fermi energy corresponds to $0 \mathrm{eV}$. The insets show results in a small energy range around the Fermi energy.

peak is created by the $5 \mathrm{~s}$ states of $\mathrm{Sn}$ with a significant contribution from $\mathrm{Pd} 4 \mathrm{p}$ states. The second one originates mainly from the mixed $\mathrm{Pd} 4 \mathrm{~d}$ and $\mathrm{Sn} 5 \mathrm{p}$ states with some contributions from Pd $4 \mathrm{~s}$ and Pd $4 \mathrm{p}$ states. The main contribution to the peak at $-4.1 \mathrm{eV}$ comes from the $4 \mathrm{~d}$ electrons of $\mathrm{Pd}$ atoms with a small hybridization of Sn $5 p$ states. The peak at -3.3 is mainly characterized by $\mathrm{Pd} 4 \mathrm{~d}$ states with much lesser contributions coming from Y 4d, Sn 4d, Sn 4p, and $\mathrm{Pd} 4 \mathrm{p}$ states. The sharpest peak at $-2.8 \mathrm{eV}$ arises from the $4 \mathrm{~d}$ electrons of $\mathrm{Pd}$ with negligible contributions from other electronic states. The last peak below the Fermi level is located at $-1.1 \mathrm{eV}$. This peak is mainly due to Pd $4 \mathrm{~d}$ states with smaller contributions coming from other electronic states. The density of states at the Fermi level $\left(\mathrm{N}\left(\mathrm{E}_{F}\right)\right)$ amounts to be 2.06 States/eV which can be compared with its experimental value of $2.23 \mathrm{State} / \mathrm{eV}$ in the work of Klimczuk et al. ${ }^{15}$ The orbital analysis of the DOS shows that mainly the Pd 4d (34\%), Y 4d (25\%), and Sn 5p (20\%) states are contributing to the total value of DOS at the Fermi level. Finally, the unoccupied DOS are mainly dominated by the $4 \mathrm{~d}$ electrons of $\mathrm{Y}$ atom. 


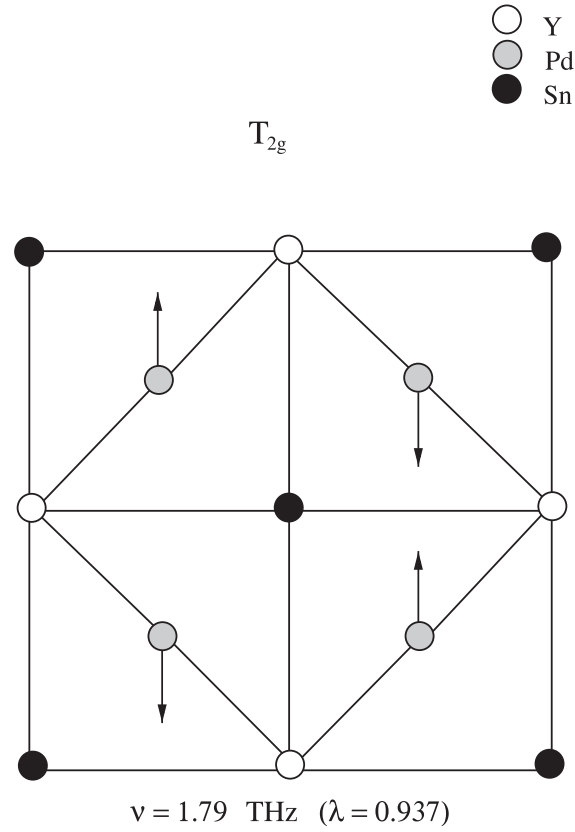

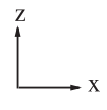

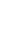

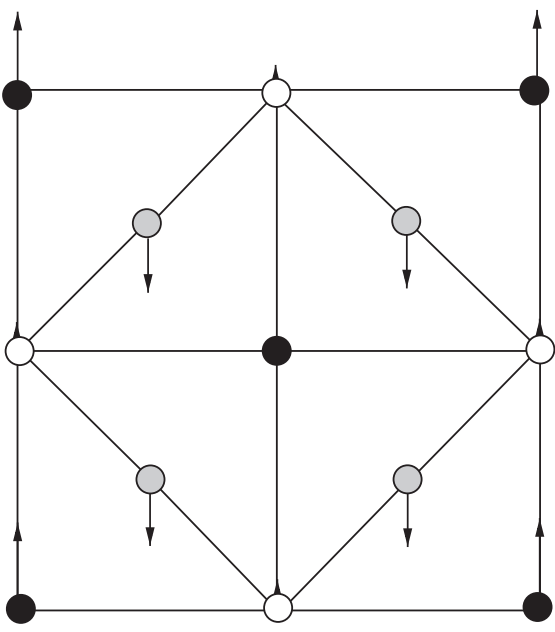

$v=3.45 \mathrm{THz} \quad(\lambda=0.001)$

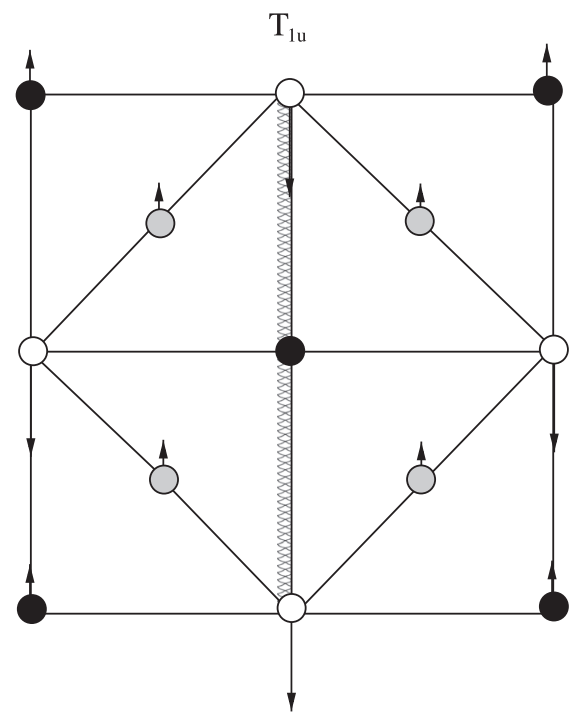

$v=4.76 \mathrm{THz} \quad(\lambda=0.001)$
FIG. 4. Eigenvectors representations of zone-centre phonon modes for $\mathrm{YPd}_{2} \mathrm{Sn}$. The electron-phonon coupling parameter for these phonon modes is also given.

\section{B. Electron-phonon interaction and superconductivity}

The primitive unit cell of the $\mathrm{YPd}_{2} \mathrm{Sn}$ Heusler structure has one formula unit (total four atoms), giving a total of 12 phonon branches, including three acoustic branches and nine optical branches. The optical phonon modes correspond to the following irreducible representations at the zone-centre:

$$
\Gamma=T_{2 g}+2 T_{1 u} .
$$

Each $\mathrm{T}$ vibration is threefold degenerate. The $\mathrm{T}_{2 g}$ mode is Raman active while the $T_{1 u}$ modes are infrared active. We have illustrated the atomic displacement pattern of these zonecentre phonon modes together with their electron-phonon coupling parameters in Fig. 4. The opposing vibrations of $\mathrm{Pd}$ atoms generate the $\mathrm{T}_{2 g}$ phonon mode at $1.79 \mathrm{THz}$. It is important to note that the largest electron-phonon coupling parameter $(\lambda=0.937)$ is found for this phonon mode. This observation is not surprising since the largest contribution to the $\mathrm{N}\left(\mathrm{E}_{F}\right)$ comes from the $\mathrm{d}$ states of Pd atoms. We have to mention that the total electron-phonon coupling parameter for this phonon mode is three times of this value due to mode degeneracy. The first $\mathrm{T}_{1 u}$ mode is due the opposing motion of $\mathrm{Sn}$ and $\mathrm{Pd}$ atoms with negligible contribution coming from $\mathrm{Y}$ atoms. The second one includes atomic vibrations of $\mathrm{Y}$ atoms against $\mathrm{Pd}$ and $\mathrm{Sn}$ atoms with maximum contribution coming from $\mathrm{Y}$ atoms due to their light mass.

In Fig. 5(a), the calculated phonon-dispersion relations are illustrated along several high-symmetry directions in the face-centered cubic zone. As can be seen, all phonon modes have positive frequencies, and there are no phonon branches with dispersions that dip towards the zero frequency. This picture confirms that the cubic Heusler $\mathrm{YPd}_{2} \mathrm{Sn}$ is dynamically stable. The phonon dispersion curves can be separated into two apparent regions: a low-frequency region between 0 and $3.7 \mathrm{THz}$ and a high-frequency region between 4.5 and $5.3 \mathrm{THz}$. A narrow gap of about $0.8 \mathrm{THz}$ between these 
(a)

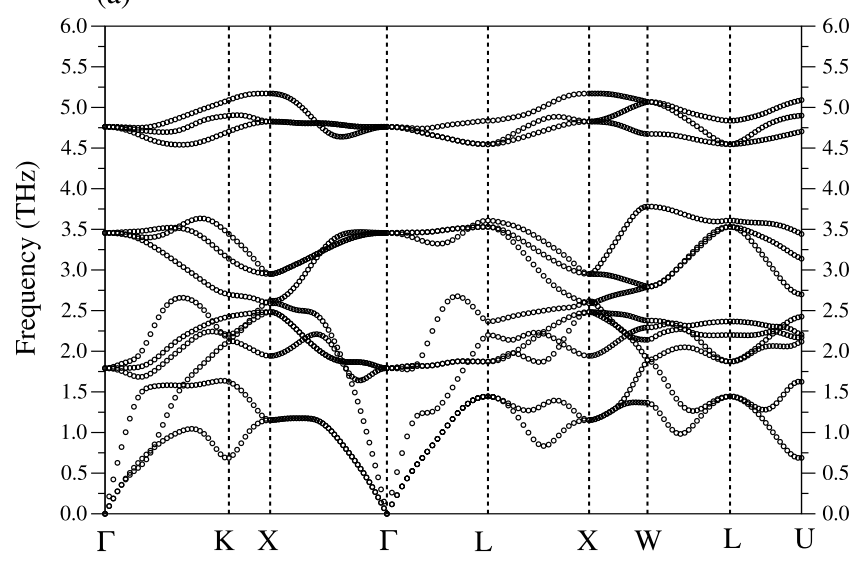

(b)

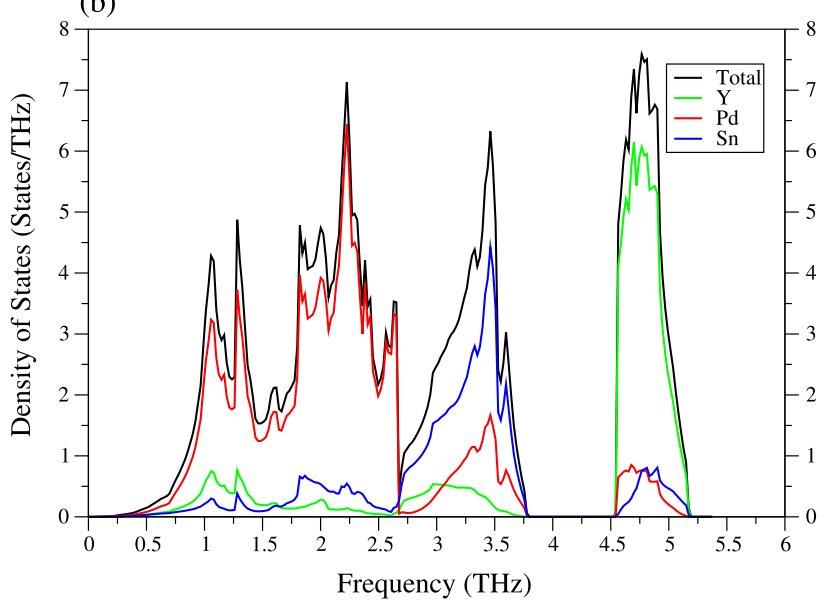

FIG. 5. (a) Calculated phonon dispersion relations for the Heusler compound $\mathrm{YPd}_{2} \mathrm{Sn}$ along symmetry lines of the face-centered cubic Brillouin zone. (b) The calculated phonon total and atom-projected density of states.

regions comes from the mass difference between the different types of atoms in the unit cell. There are nine phonon branches extending up to $3.7 \mathrm{THz}$ in the low-frequency region. All phonon branches in this region exhibit considerable amount of dispersion. The most striking feature of our phonon spectrum is the pronounced minimum of transverse acoustic branch (TA) along $\Gamma-\mathrm{K}, \mathrm{L}-\mathrm{X}, \mathrm{W}-\mathrm{L}$, and L-U symmetry directions. We strongly believe that these phonon anomalies play an important role in developing superconductivity in the Heusler compound $\mathrm{YPd}_{2} \mathrm{Sn}$ since a similar observation has been made for several superconductors. ${ }^{27-31}$ There are three optical branches in the high-frequency region of the phonon spectrum. These phonon branches are less dispersive than the optical phonon branches in the lowfrequency region. The nature of the phonon dispersion relations can be understood more clearly by the total and partial phonon DOS in Fig. 5(b). The partial DOS illustrates a dominance of $\mathrm{Pd}$ atoms in the low energy range up to $2.7 \mathrm{THz}$. The intermediate frequency region between 2.7 and $3.7 \mathrm{THz}$ is mainly dominated by the vibrations of $\mathrm{Sn}$ atoms with smaller contributions coming from the vibrations of remaining atoms. Above the phonon band gap region, the vibrations of $\mathrm{Y}$ atoms are very dominant due to their lighter atom mass than the other two atoms.
As we have pointed out above, the most interesting feature of the phonon dispersion relations for $\mathrm{YPd}_{2} \mathrm{Sn}$ is the anomalous behaviour exhibited by the lower acoustic branch along the $\Gamma$-K symmetry direction. In order to find a relationship between the anomalous phonon dispersion and electronphonon interaction, we have calculated the mode-dependent electron-phonon coupling parameter $\lambda_{\mathbf{q}, j}$ for the lowfrequency phonon branches. Exciting features can be noted along the two main symmetry directions [110] and [111] in Fig. 6. First, along the [110] direction, the $\mathrm{T}_{2 g}$ mode splits into three phonon branches: two transverse optical branches $\left(\mathrm{TO}_{1}\right.$ and $\left.\mathrm{TO}_{2}\right)$ and one longitudinal optical (LO) branch. Along [111] direction, transverse branches are doubly degenerate. The electron-phonon coupling parameters of these optical branches decreases rapidly with increasing $\mathbf{q}$ wave vector along both the symmetry directions. Thus, these phonon branches make a large contribution to electron-phonon interaction in $\mathrm{YPd}_{s} \mathrm{Sn}$ only close to zone-centre. Second, the $\mathrm{TA}_{2}$ branch lies below the $\mathrm{TA}_{1}$ branch in the some part of $\Gamma-\mathrm{K}$ direction. This phonon anomaly is found to occur between $\mathbf{q}=\frac{2 \pi}{a}(0.10,0.10,0.00)$ and $\mathbf{q}=\frac{2 \pi}{a}(0.25,0.25,0.00)$. As a consequence of this phonon anomaly, the electron-phonon coupling parameter of this branch hardens rapidly with increasing wave vector up to $\mathbf{q}=\frac{2 \pi}{a}(0.25,0.25,0.00)$. In particular, the most notable feature is that the electron-phonon coupling parameter of the $\mathrm{TA}_{2}$ branch reaches a peak value of 1.20 at $\mathbf{q}=\frac{2 \pi}{a}(0.225,0.225,0.000)$. Third, the branch $\mathrm{TA}_{1}$ starts to show a negative dispersion (phonon anomaly) beyond $\quad \mathbf{q}_{0}=\frac{2 \pi}{a}(0.575,0.575,0.00)$. Thus, this branch acquires a clear "dip" close to the K zone-boundary. Due to this phonon anomaly, the electron-phonon coupling parameter of this phonon branch increases with increasing $\mathbf{q}$ wave vector beyond $\mathbf{q}_{0}$ up to the $K$ point. The peak value of electron-phonon coupling parameter for this phonon branch is found to be 1.12 at the $\mathrm{K}$ point. Thus, it will be interesting to analyze the atomic displacement pattern of $\mathrm{TA}_{1}$ phonon mode at the $\mathrm{K}$ point. Fig. 7 presents the eigenvectors representation of this phonon mode. As can be seen from this figure, this phonon mode is mainly dominated by the vibrations of $\mathrm{Pd}$ atoms with some contributions coming from other atoms in the unit cell. Different from the [110] direction, the doubly degenerate TA branch behaves normally and shows a positive dispersion with increasing $\mathbf{q}$ wave vector along the [111] direction. However, the LA branch makes a larger contribution to the electron-phonon interaction than the TA branches. In particular, the electron-phonon coupling parameter of LA branch takes its largest value of 0.63 at $\mathbf{q}=\frac{2 \pi}{a}(0.20,0.20,0.20)$.

In order to sight the strengths with which different modes of the atomic motion couple to electrons, and thus are skillful in affecting superconductivity in $\mathrm{YPd}_{2} \mathrm{Sn}$ most, we have displayed the Eliashberg spectral function together with the variation of the electron-phonon coupling parameter with increasing frequency $\lambda(\omega)$ in Fig. 8. By integrating the Eliashberg spectral function using Eq. (3), the average electron-phonon coupling parameter is found to be $\lambda=0.99$. A comparison of the Eliashberg spectral function $\alpha^{2} \mathrm{~F}(\omega)$ with the phonon density of states (see Fig. 5(b)) shows that the features in the electron-phonon spectral function are 
(a)
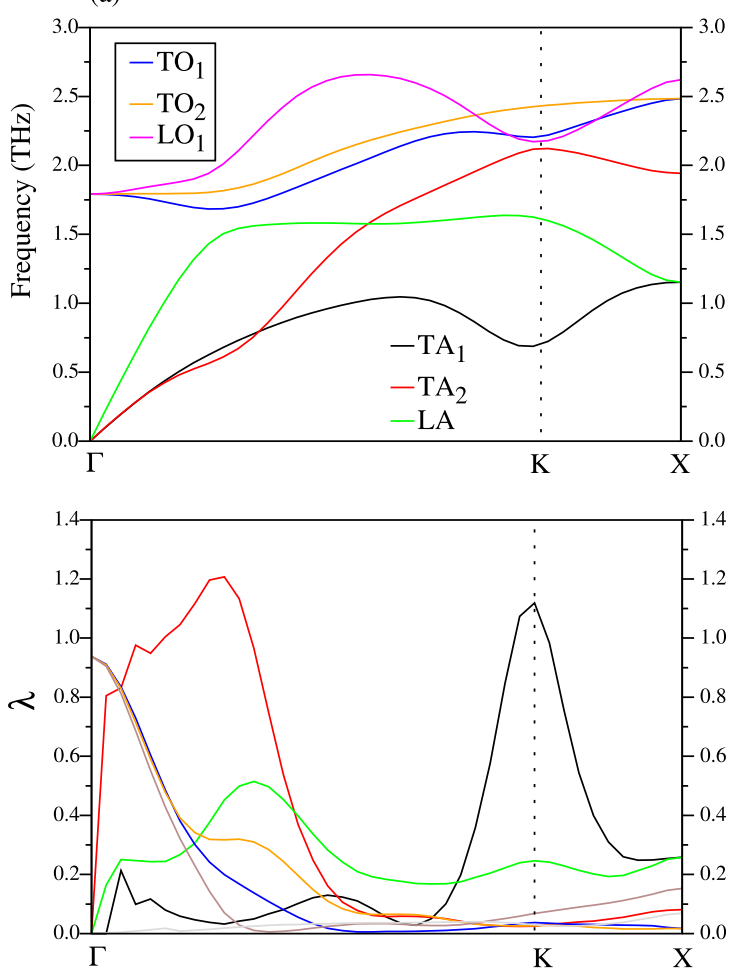

(b)
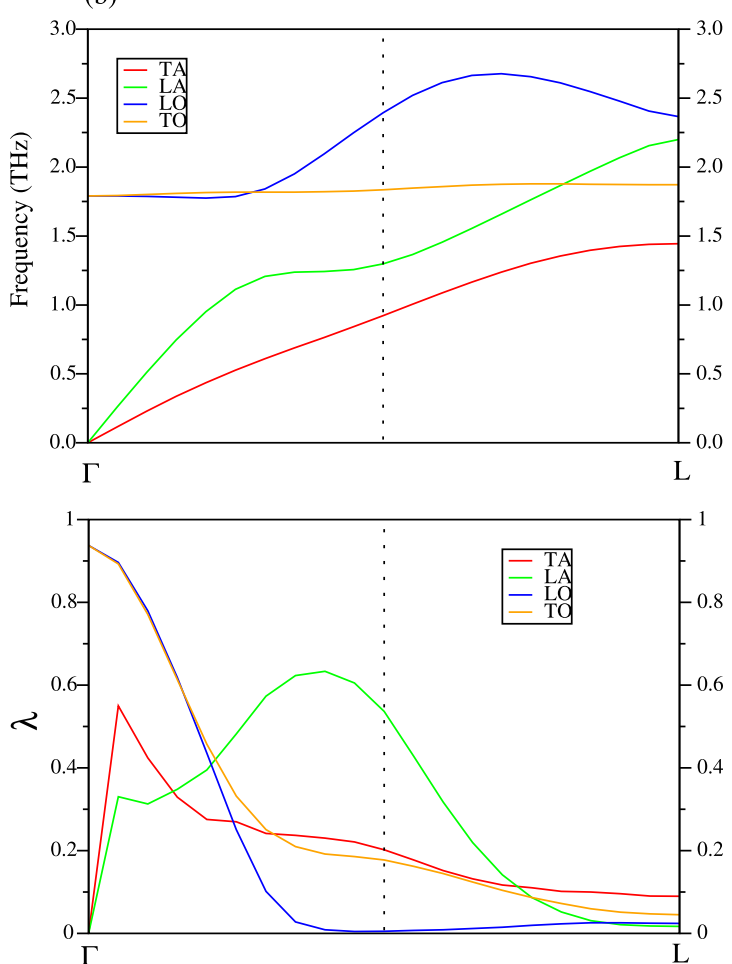

FIG. 6. The dispersion of the lowest six phonon branches with their corresponding electron-phonon coupling parameter (a) along the [110] direction and (b) along the [111] direction.

considerably decreased relative to the corresponding features in the phonon density of states above the phonon band gap region. Thus, we can say that phonon branches above the gap region make very small contribution to the average electron-phonon coupling parameter (their contribution is within 4\%). Such small contribution from high frequency phonons can be connected with the factor $\frac{1}{\omega}$ in Eq. (3).
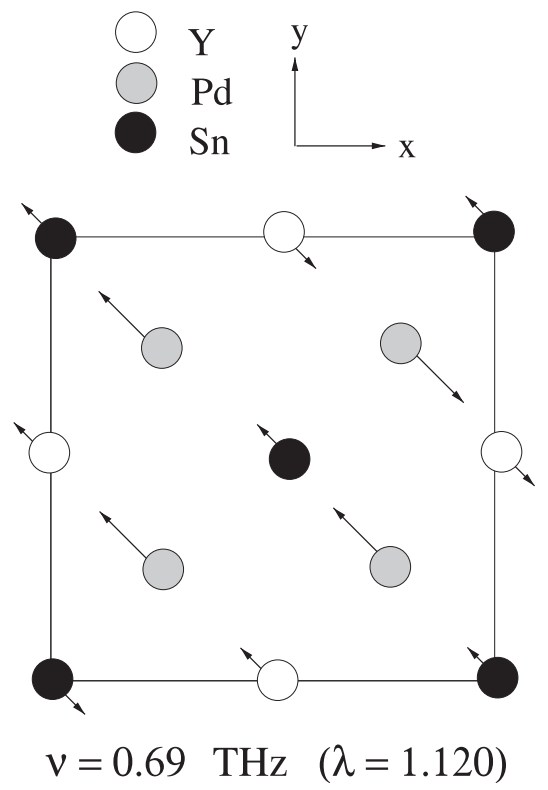

FIG. 7. Eigenvectors representations of first transverse acoustic phonon mode $\left(\mathrm{TA}_{1}\right)$ at the $\mathrm{K}$ point for $\mathrm{YPd}_{2} \mathrm{Sn}$. The electron-phonon coupling parameter for this phonon is also presented.
However, phonon modes below $2.7 \mathrm{THz}$ contribute about $88 \%$ towards $\lambda$. Our partial phonon density of states shows that the Pd-related phonon modes exist below $2.7 \mathrm{THz}$. This is an expected result due to considerable contribution of $\mathrm{Pd} \mathrm{d}$ states to $\mathrm{N}\left(\mathrm{E}_{F}\right)$.

With $\lambda$ computed, the transition temperature $\left(\mathrm{T}_{C}\right)$ for $\mathrm{YPd}_{2}$ Sn can be calculated from the Allen-Dynes formula ${ }^{24,25}$

$$
T_{C}=\frac{\omega_{\ln }}{1.2} \exp \left(-\frac{1.04(1+\lambda)}{\lambda-\mu^{*}(1+0.62 \lambda)}\right),
$$

where the logarithmically averaged phonon frequency $\omega_{\ln }$ is given as

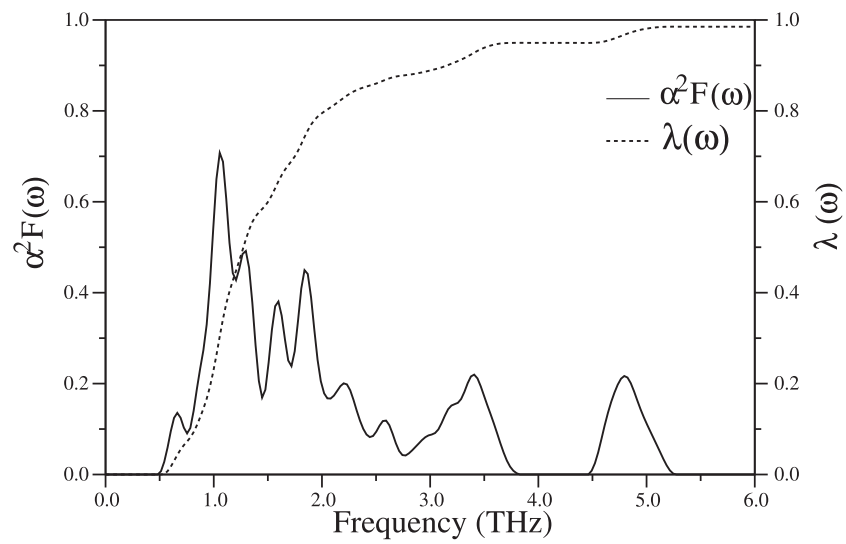

FIG. 8. The calculated electron-phonon spectral function $\alpha^{2} \mathrm{~F}(\omega)$ (left axis) and the variation of the electron-phonon coupling parameter (right axis) with increasing frequency $\lambda(\omega)$. 


$$
\omega_{\ln }=\exp \left(2 \lambda^{-1} \int_{0}^{\infty} \frac{d \omega}{\omega} \alpha^{2} F(\omega) \ln \omega\right)
$$

Inserting the calculated value of $\lambda$ into Eq. (5), the value of $\omega_{\ln }$ is found to be $1.47 \mathrm{THz} . \mu^{*}$ in Eq. (4) is the screened Coulomb pseudopotential parameter, which takes a value between 0.1 and $0.16 .^{24,25}$ Using the Allen-Dynes formula and taking typical values of $\mu^{*}=0.10,0.11,0.12,0.13,0.14$, 0.15 , and 0.16 , we obtain $\mathrm{T}_{C}=4.81,4.57,4.32,4.11,3.89$, 3.66 , and 3.45 , respectively. The average of these values is $4.12 \mathrm{~K}$, which is in acceptable agreement with the experimental values of $3.74,{ }^{9} 4.55,{ }^{12} 5.04,{ }^{13}$ and $4.7 \mathrm{~K} .{ }^{15}$

The calculated values of the superconducting state parameters for $\mathrm{YPd}_{2} \mathrm{Sn}$ are compared with their corresponding experimental values in Table II. In general, our results are in qualitative agreement with available experimental results. ${ }^{9,10,12,13,15}$ In particular, the calculated value of $\mathrm{N}\left(\mathrm{E}_{F}\right)$ is slightly lower than its experimental value of 2.23 (Ref. 15) while the calculated value of $\lambda$ is higher than its experimental value of $0.70 .{ }^{15}$ When making this comparison, it should be noted that the computed value of $\mathrm{N}\left(\mathrm{E}_{F}\right)$ comes directly from our band structure results, while the experimentally quoted value is estimated from the measured Sommerfeld coefficient of the specific heat and the estimate of $\lambda$. Also, our calculated value of $\lambda=0.96$ is higher than the experimental value of 0.70 . We have used the quasi-static approximation $^{32}$ to calculate the Debye temperature $(\Theta)$ of $\mathrm{YPd}_{2} \mathrm{Sn}^{33,34}$ The value of $\Theta$ is found to be $199 \mathrm{~K}$, which is in an excellent agreement with the recent experimental value of $210 \mathrm{~K}$ in the work of Klimczuk et al. ${ }^{15}$ The electron-phonon coupling constant $\lambda$ also enters the electronic specific heat coefficient $\gamma$, which is given as

$$
\gamma=\frac{1}{3} \pi^{2} k_{B}^{2} N\left(E_{F}\right)(1+\lambda)
$$

From the above equation, the value of $\gamma$ is obtained to be $9.6 \mathrm{~mJ} \mathrm{~mol}^{-1} \mathrm{~K}^{-2}$, which compares very well with its experimental value of $9.2 \mathrm{~mJ} \mathrm{~mol}^{-1} \mathrm{~K}^{-2} \cdot{ }^{15}$ Finally, the BCS energy gap $\left(\Delta=1.76 \mathrm{k}_{B} \mathrm{~T}_{C}\right)$ is found to be $0.49 \mathrm{~K}$, which can compared with the corresponding experimental value of $0.83 \mathrm{~K}$ in the work of Klimczuk et al. ${ }^{15}$ The agreement between our results and recent experimental results ${ }^{15}$ signs the good quality of our GGA calculations. As a result, our calculations indicate that the Heusler compound $\mathrm{YPd}_{2} \mathrm{Sn}$ is a conventional phonon-mediated superconductor with the strong electron-phonon coupling strength of 0.99 .

TABLE II. The comparison of superconducting state parameters for $\mathrm{YPd}_{2} \mathrm{Sn}$. We have used $\Delta=1.76 \mathrm{k}_{B} \mathrm{~T}_{C}$.

\begin{tabular}{lccccccc}
\hline \hline Source & $\begin{array}{c}\mathrm{N} \\
\left(\mathrm{E}_{F}\right)\end{array}$ & $\lambda$ & $\begin{array}{c}\omega_{\mathrm{ln}} \\
(\mathrm{K})\end{array}$ & $\begin{array}{c}\Theta \\
(\mathrm{K})\end{array}$ & $\begin{array}{c}\mathrm{T}_{C} \\
(\mathrm{~K})\end{array}$ & $\begin{array}{c}\gamma \\
\left(\frac{\mathrm{mJ}}{\mathrm{mol} \mathrm{K}^{2}}\right)\end{array}$ & $\begin{array}{c}\Delta \\
(\mathrm{meV})\end{array}$ \\
\hline This work $^{2}$ & 2.06 & 0.96 & 70.96 & 199 & 4.12 & 9.5 & 0.49 \\
Experimental $^{9,10}$ & & & & 165 & 3.72 & & \\
Experimental $^{12}$ & & & & & 4.55 & & \\
Experimental $^{13}$ & & & & & 5.04 & & \\
Experimental $^{15}$ & 2.23 & 0.70 & & 210 & 4.7 & 9.2 & 0.83 \\
\hline \hline
\end{tabular}

\section{SUMMARY}

We have studied the static and electronic properties of the Heusler compound $\mathrm{YPd}_{2} \mathrm{Sn}$ applying density functional theory within a generalised gradient approximation. Our results compare very well with existing experimental results for lattice constant and bulk modulus. Our electronic calculations validate the metallic nature of this compound. From the analysis of the total and partial electronic density of states, we have observed that the states around the Fermi level are contributed by Pd 4d, Y 4d, and Sn 5p states with much smaller contribution from other electronic states.

Using our calculated lattice constant and electronic structure, lattice dynamics of $\mathrm{YPd}_{2} \mathrm{Sn}$ has been studied by employing a linear response approach based on density functional perturbation theory. This compound is dynamically stable, as no instabilities in the phonon dispersion curves have been found. However, a striking feature in the phonon spectrum of this material is the pronounced minimum of transverse acoustic branches along the symmetry direction [110]. From the analysis of phonon density of states, we have found that Pd-related vibrations are dominant in the low frequency region up to $2.7 \mathrm{THz}$. On the other hand, Sn-related vibrations are strong in the middle frequency region between 2.7 and $3.7 \mathrm{THz}$. Above the phonon band gap region, Y-related vibrations are dominant due to the smaller mass of $\mathrm{Y}$ atom.

We have presented a numerically accurate correlation between phonon anomalies and the electron-phonon coupling parameter by studying electron-phonon interaction in this material. Our electron-phonon interaction results show that the electron phonon coupling parameters of the acoustic branches $\left(\mathrm{TA}_{1}\right.$ and $\left.\mathrm{TA}_{2}\right)$ reach their peak values of 1.12 and 1.20 , respectively, along the $\Gamma-\mathrm{K}$ direction where phonon anomalies are observed.

The phonon spectrum and the electron-phonon matrix elements are used to calculate the Eliashberg function from which the superconducting properties can be obtained. The deviation of the shape of Eliashberg function from that of phonon density of states confirms that the phonon modes related to Pd-vibrations play a very important role in the process of scattering of electrons. From the Eliashberg function, the average electron-phonon coupling parameter is calculated to be 0.99 , indicating reasonably strong interaction between electrons and phonons in this compound. Using the calculated the values of $\lambda$ and $\omega_{\ln }$, the transition temperature $\left(\mathrm{T}_{C}\right)$ calculated from the Allen-Dynes formula is found to be $4.12 \mathrm{~K}$, which compares well with the recent experimental value of $4.7 \mathrm{~K}$.

${ }^{1}$ M. Julliere, Phys. Lett. A 54, 225 (1975).

${ }^{2}$ R. A. de Groot, F. M. Mueller, P. G. van Engen, and K. H. J. Buschow, Phys. Rev. Lett. 50, 2024 (1983).

${ }^{3}$ K. Mastronardi, D. Young, C.-C. Wang, P. Khalifah, R. J. Cava, and A. P. Ramirez, Appl. Phys. Lett. 74, 1415 (1999).

${ }^{4}$ S. A. Wolf, D. D. Awschalom, R. A. Buhrman, J. M. Daughton, S. von Molnar, M. L. Roukes, A. Y. Chtchelkanova, and D. M. Treger, Science 294, 1488 (2001).

${ }^{5}$ M. H. Jung, T. Yoshino, S. Kawasaki, T. Pietrus, Y. Bando, T. Suemitsu, M. Sera, and T. Takabatake, J. Appl. Phys. 89, 7631 (2001). 
${ }^{6}$ C. Felser, G. H. Fecher, and B. Balke, Angew. Chem., Int. Ed. 46, 668 (2007).

${ }^{7}$ T. Graf, C. Felser, and S. S. P. Parkin, Prog Solid State Chem. 39, 1 (2011).

${ }^{8}$ I. Zutic, J. Fabian, and S. Das Sarma, Rev. Mod. Phys. 76, 323 (2004).

${ }^{9}$ M. Ishikawa, J.-L. Jorda, and A. Junod, in Superconductivity in d- and f-Band Metals, edited by W. Buckel and W. Weber (Kernforschungszentrum, Karlsruhe, 1982), p. 141.

${ }^{10}$ M. J. Johnson and R. N. Shelton, Solid State Commun. 52, 839 (1984).

${ }^{11}$ S. K. Malik, A. M. Umarji, and G. K. Shenoy, Phys. Rev. B 31, 6971 (1985).

${ }^{12}$ S. K. Malik, A. M. Umarji, and G. K. Shenoy, Phys. Rev. B 32, 4426 (1985).

${ }^{13}$ R. N. Shelton, L. S. Hausermann-Berg, M. J. Johnson, P. Klavins, and H. D. Yang, Phys. Rev. B 34, 199 (1986).

${ }^{14}$ C. Höting, H. Eckert, T. Langer, I. Schellenberg, and R. Pöttgen, J. Solid State Chem. 190, 216 (2012).

${ }^{15}$ T. Klimczuk, C. H. Wang, K. Gofryk, F. Ronning, J. Winterlik, G. H. Fecher, J.-C. Griveau, E. Colineau, C. Felser, J. D. Thompson, D. J. Safarik, and R. J. Cava, Phys. Rev. B 85, 174505 (2012).

${ }^{16}$ H. Saadaoui, T. Shiroka, A. Amato, C. Baines, H. Luetkens, E. Pomjakushina, V. Pomjakushin, J. Mesot, M. Pikulski, and E. Morenzoni, Phys. Rev. B 85, 094518 (2013).

${ }^{17}$ P. Giannozzi, S. Baroni, N. Bonini, M. Calandra, R. Car, C. Cavazzoni, D. Ceresoli, G. L. Chiarotti, M. Cococcioni, I. Dabo, A. D. Corso, S. de Gironcoli, S. Fabris, G. Fratesi, R. Gebauer, U. Gerstmann, C. Gougoussis, A. Kokalj, M. Lazzeri, L. Martin-Samos, N. Marzari, F. Mauri, R. Mazzarello, S. Paolini, A. Pasquarello, L. Paulatto, C. Sbraccia,
S. Scandolo, G. Sclauzero, A. P. Seitsonen, A. Smogunov, P. Umari, and R. M. Wentzcovitch, J. Phys.: Condens. Matter 21, 395502 (2009).

${ }^{18}$ W. Kohn and L. J. Sham, Phys. Rev. 140, A1133 (1965).

${ }^{19}$ J. P. Perdew, K. Burke, and M. Erzerhof, Phys. Rev. Lett. 77, 3865 (1996).

${ }^{20}$ S. Goedecker, M. Teter, and J. Hutter, Phys. Rev. B 54, 1703 (1996).

${ }^{21}$ C. Hartwigsen, S. Goedecker, and J. Hutter, Phys. Rev. B 58, 3641 (1998).

${ }^{22}$ H. J. Monkhorst and J. D. Pack, Phys. Rev. B 13, 5188 (1976).

${ }^{23}$ G. M. Eliashberg, Sov. Phys. JETP 11, 696 (1960).

${ }^{24}$ P. B. Allen, Phys. Rev. B 6, 2577 (1972).

${ }^{25}$ P. B. Allen and R. C. Dynes, Phys. Rev. B 12, 905 (1975).

${ }^{26}$ F. D. Murnaghan, Proc. Nat. Acad. Sci. U. S. A. 30, 244 (1944).

${ }^{27}$ E. I. Isaev, R. Ahuja, S. I. Simak, A. I. Lichtenstein, Yu. Kh. Vekilov, B. Johansson, and I. A. Abrikosov, Phys. Rev. B 72, 064515 (2005).

${ }^{28}$ E. I. Isaev, S. I. Simak, I. A. Abrikosov, R. Ahuja, Yu. Kh. Katsnelson, A. I. Lichtenstein, and B. Johansson, J. Appl. Phys. 101, 123519 (2007).

${ }^{29}$ S. Bağcl, S. Duman, H. M. Tütüncü, and G. P. Srivastava, Phys. Rev. B 78, 174504 (2008)

${ }^{30}$ S. Bağc1, H. M. Tütüncü, S. Duman, and G. P. Srivastava, Phys. Rev. B 81, 144507 (2010).

${ }^{31}$ H. M. Tütüncü, S. Bağcl, G. P. Srivastava, and A. Akbulut, J. Phys.: Condens. Matter 24, 455704 (2012).

${ }^{32}$ Y. Wang, J. J. Wang, H. Zhang, V. R. Manga, S. L. Shang, L.-Q. Chen, and Z.-K. Liu, J. Phys.: Condens. Matter 22, 225404 (2010).

${ }^{33}$ S. Baroni, P. Giannozzi, and E. Isaev, Mineral. Geochem. 71, 39-57 (2010).

${ }^{34}$ H. M. Tütüncü and G. P. Srivastava, Philos. Mag. 93, 4469 (2013). 